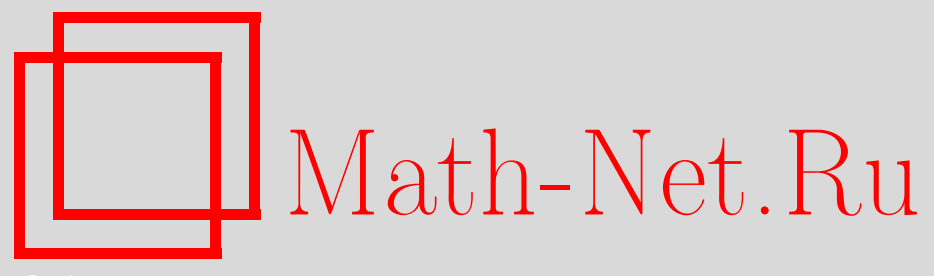

С. И. Калмыков, Об асимптотически точном неравенстве марковского типа для тригонометрических и алгебраических многочленов, Матем. заметки, 2015, том 98, выпуск 2, 303-307

DOI: https://doi.org/10.4213/mzm10720

Использование Общероссийского математического портала Math-Net.Ru подразумевает, что вы прочитали и согласны с пользовательским соглашением http: //www . mathnet.ru/rus/agreement

Параметры загрузки:

IP: 3.82 .47 .9

26 апреля 2023 г., 14:15:04

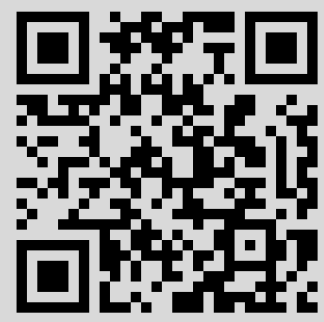




\section{Об асимптотически точном неравенстве марковского типа для тригонометрических и алгебраических многочленов}

\section{С. И. Калмыков}

Введение. Неравенство Маркова для производной алгебраического многочлена на отрезке $[-1,1]$ является одним из самых известных полиномиальных неравенств (см., например, [1; теорема 5.1.8]). Также имеет место его аналог для тригонометрического многочлена на отрезке меньше, чем период [1; 5.E.14, с. 238 и 5.Е.19, с. 243]. В 2001 г. Тотик, используя метод полиномиальных прообразов, доказал асимптотически точное неравенство марковского типа для алгебраических полиномов на нескольких отрезках [2; теорема 4.1]. А в работе [3; теорема 4.1, следствия 4.2, 4.3] асимптотически точные неравенства указанного типа были получены для тригонометрических многочленов на нескольких отрезках и для алгебраических многочленов на нескольких дугах окружности. Случай одной дуги был рассмотрен в [4; с. 225]. В недавней работе [5], посвященной алгебраическим многочленам, компакт, состоящий из нескольких отрезков, был заменен более общим компактом, а именно, компактом, имеющим точку такую, что выполнено условие, подобное условию (1), сформулированному ниже. Целью настоящей работы является аналогичное обобщение для тригонометрических многочленов на $2 \pi$-периодическом замкнутом подмножестве $\mathscr{E}$ вещественной оси $\mathbb{R}$ и, как следствие, для алгебраических многочленов на компактном подмножестве единичной окружности.

Обозначим через $P_{n}$ и $T_{n}$ алгебраический и тригонометрический многочлены со степенями не выше $n$ соответственно, а через $\|\cdot\|_{K}$ sup-норму на множестве $K$, и пусть $E=$ $\mathscr{E} \cap[-\pi, \pi]$, а $\Gamma_{E}=\left\{z: z=e^{i t}, t \in E\right\}$. Так как очевидно, что $\left\|T_{n}\right\|_{\mathscr{E}}=\left\|T_{n}\right\|_{E}$, будем использовать второй вариант. Точку а назовем правой концевой точкой компакта $E$, если найдется число $\rho>0$ такое, что

$$
[a-2 \rho, a] \subset E \quad \text { и } \quad(a, a+2 \rho) \cap E \neq \varnothing .
$$

Аналогично определяется левая концевая точка. Не теряя общности, далее по тексту будем считать, что $a \in(-\pi, \pi)$ и что $a$ - правая концевая точка. Также в этом случае будем говорить, что $e^{i a}-$ концевая точка множества $\Gamma_{E}$.

Для того чтобы сформулировать результаты, нам понадобятся сведения из теории потенциала (см. например, [6], [7]). В частности, для равновесной меры компактного множества $K \subset \mathbb{R}$ (или $\mathbb{T}=\{|z|=1\})$, имеющего положительную емкость $(\operatorname{cap}(K)>0)$, введем обозначение $\nu_{K}$. Эта мера абсолютно непрерывна во внутренних точках (размерности один) компакта $K$, и через $\omega_{K}(u)$ обозначим ее плотность по мере Лебега, т.е. $\omega_{K}(u)=d \nu_{K}(u) / d u$. Если $u_{0}$ - концевая точка компакта $K$, тогда около точки $u_{0}$ плотность $\omega_{K}$ ведет себя так же, как $1 / \sqrt{\left|u-u_{0}\right|}$ (см. [5; лемма 2.1], где при необходимости нужно перейти от компактов на $\mathbb{R}$ к компактам на $\mathbb{T}$, и [8; утверждение 2.2 , с. 119$])$. Ключевую роль в работе играет величина

$$
\Omega\left(\Gamma_{E}, e^{i a}\right):=\lim _{t \rightarrow a-0} \omega_{E}\left(e^{i t}\right)\left|e^{i t}-e^{i a}\right|^{1 / 2} .
$$

Непосредственно из теоремы IV.1.6,е в книге [6] следует монотонность этой величины: если для компактов $K$ и $S$ выполнено (1) и, кроме того, $K \subset S$, то $\Omega\left(\Gamma_{S}, e^{i a}\right) \leqslant \Omega\left(\Gamma_{K}, e^{i a}\right)$.

Множество $[-\pi, \pi] \backslash E$ состоит из конечного или счетного числа интервалов, открытых в $[-\pi, \pi]$ :

$$
[-\pi, \pi] \backslash E=\bigcup_{j=0}^{\infty} I_{j}
$$

Работа выполнена при поддержке Российского научного фонда (грант № 14-11-00022).

DOI: $10.4213 / \mathrm{mzm} 10720$ 
(если $E$ состоит из конечного числа отрезков, то бесконечное объединение заменяется конечным). Для определенности будем считать, что $I_{0}$ содержит $(a, a+2 \rho)$. Далее, для $m \geqslant 0$ рассмотрим множество

$$
\begin{aligned}
E_{m}^{+}=[-\pi, \pi] \backslash\left(\bigcup_{j=0}^{m-2} I_{j}\right)= & \bigcup_{j=1}^{m^{\prime}}\left[a_{j, m^{\prime}}, b_{j, m^{\prime}}\right], \\
& a_{1, m^{\prime}} \leqslant b_{1, m^{\prime}}<a_{2, m^{\prime}} \leqslant b_{2, m^{\prime}}<\cdots<a_{m^{\prime}, m^{\prime}} \leqslant b_{m^{\prime}, m^{\prime}}=b_{0, m^{\prime}} .
\end{aligned}
$$

Очевидно, что $E_{m}^{+}$содержит $E$ и удовлетворяет условию (1). Далее, $\Gamma_{E_{m}^{+}}=\left\{z: z=e^{i t}\right.$, $\left.t \in E_{m}^{+}\right\}$. Если для некоторого $j a_{j, m^{\prime}}=b_{j, m^{\prime}}$, то заменим этот вырожденный отрезок отрезком

$$
\left[a_{j, m^{\prime}}-\lambda_{m}, a_{j, m^{\prime}}+\lambda_{m}\right] \cap[-\pi, \pi],
$$

где $\lambda_{m}<1 / m$ выбирается настолько малым, чтобы все еще выполнялось условие (1). За получившимся множеством сохраним обозначение $E_{m}^{+}$.

Так как $E_{m}^{+}$состоит из конечного числа отрезков, то (см. [3; лемма 3.5$]$ и [9; лемма 4.1])

$$
\omega_{\Gamma_{E_{m}^{+}}}\left(e^{i t}\right)=\frac{1}{2 \pi} \frac{\prod_{j=0}^{m^{\prime}-1}\left|e^{i t}-e^{i \tau_{j}}\right|}{\sqrt{\prod_{j=1}^{m^{\prime}}\left|e^{i t}-e^{i a_{j, m^{\prime}}}\right|\left|e^{i t}-e^{i b_{j, m^{\prime}}}\right|}}, \quad t \in \operatorname{Int} E_{m}^{+},
$$

где числа $e^{i \tau_{j}}$ выбраны таким образом, что

$$
\int_{b_{k, m^{\prime}}}^{a_{k+1, m^{\prime}}} \frac{\prod_{j=0}^{m^{\prime}-1}\left(e^{i t}-e^{i \tau_{j}}\right)}{\sqrt{\prod_{j=1}^{m^{\prime}}\left(e^{i t}-e^{i a_{j, m^{\prime}}}\right)\left(e^{i t}-e^{i b_{j, m^{\prime}}}\right)}} d t=0, \quad k=0,1, \ldots, m^{\prime}-1 .
$$

Под $\sqrt{\prod_{j=1}^{m^{\prime}}\left(e^{i t}-e^{i a_{j, m^{\prime}}}\right)\left(e^{i t}-e^{i b_{j, m^{\prime}}}\right)}$ понимается регулярная ветвь в области $\overline{\mathbb{C}} \backslash \Gamma_{E_{m}^{+}}$.

Нам также понадобится частный случай результата Анкона из [10]: пусть $\Gamma_{E} \subset \mathbb{T}-$ компактное множество положительной емкости. Тогда для любого $m$ существует регулярное (в смысле задачи Дирихле в $\left.\overline{\mathbb{C}} \backslash \Gamma_{E}\right)$ компактное множество $\Gamma_{E_{m}^{-}} \subset \Gamma_{E}$ такое, что

$$
\operatorname{cap}\left(\Gamma_{E}\right) \leqslant \operatorname{cap}\left(\Gamma_{E_{m}^{-}}\right)+\frac{1}{m} .
$$

Мы можем считать, что и соответствующее множество $E_{m}^{-}$удовлетворяет условию (1), т.е. $e^{i a}$ - концевая точка множества $\Gamma_{E_{m}^{-}}$.

Лемма 1. Имеем $\Omega\left(\Gamma_{E_{m}^{ \pm}}, e^{i a}\right) \rightarrow \Omega\left(\Gamma_{E}, e^{i a}\right)$ nри $m \rightarrow \infty$.

ДокАЗАТЕльство в целом повторяет доказательство утверждения 2.3 работы [5] с заменой множеств $E_{m}^{ \pm}$на $\Gamma_{E_{m}^{ \pm}}$, где на этот раз использована $*$-слабая сходимость равновесных мер $\nu_{\Gamma_{E_{m}^{ \pm}}^{ \pm}}$к $\nu_{\Gamma_{E}}$ (cp. [5; лемма 2.2]).

Лемма 2 [6; следствие VI.3.6]. Пусть $J_{1}$ и $J_{2}-$ два непересекающихся компакта, лежашие на отрезке $J$. Тогда существуют число $c, 0<c<1$, и алгебраические полиномы $R_{d}$, $\operatorname{deg} R_{d} \leqslant d$ (для всех достаточно больших $\left.d\right)$ такие, что

$$
\begin{aligned}
\left|R_{d}(x)\right| & \leqslant c^{d}, & & x \in J_{1}, \\
\left|R_{d}(x)-1\right| & \leqslant c^{d}, & & x \in J_{2}, \\
0 \leqslant R_{d}(x) & \leqslant 1, & & x \in J .
\end{aligned}
$$




\section{Основные результаты.}

Теорема 1. Для тригонометрического полинома $T_{n}$ справедливо неравенство

$$
\left\|T_{n}^{\prime}\right\|_{[a-\rho, a]} \leqslant(1+o(1)) n^{2} 8 \pi^{2} \Omega\left(\Gamma_{E}, e^{i a}\right)^{2}\left\|T_{n}\right\|_{E} .
$$

Это неравенство точное, так как существует последовательность полиномов $T_{n} \not \equiv$, $n=1,2, \ldots$, maкux, чmо

$$
\left|T_{n}^{\prime}(a)\right| \geqslant(1-o(1)) n^{2} 8 \pi^{2} \Omega\left(\Gamma_{E}, e^{i a}\right)^{2}\left\|T_{n}\right\|_{E} .
$$

Величина о(1) равномерно по $T_{n}$ стремится $к$ нулю при $n \rightarrow \infty$.

ДоказАтельство. Если $E$ состоит из конечного числа отрезков, то все сводится к теореме 4.1 работы [3]. Далее, следуя работе [5], докажем (5) сначала в случае, когда $E$ компакт, регулярный в смысле задачи Дирихле в $\overline{\mathbb{C}} \backslash E$. Зафиксируем $\varepsilon>0$. Тогда существует тригонометрический полином $Q_{\varepsilon n}$ степени не выше $\varepsilon n$, удовлетворяющий условиям

$$
\begin{array}{rlrl}
1-e^{-n \tau} \leqslant Q_{n \varepsilon}(t) & \leqslant 1, & & t \in[a-\rho, a], \\
0 \leqslant Q_{n \varepsilon}(t) \leqslant 1, & t \in[-\pi, \pi], & \\
\left|Q_{n \varepsilon}(t)\right| & \leqslant e^{-n \tau}, & & t \in[-\pi, \pi] \backslash\left(a-\frac{3 \rho}{2}, a+\frac{3 \rho}{2}\right) .
\end{array}
$$

Существование тригонометрического многочлена с указанными свойствами вытекает из леммы 2. Действительно, пусть $t_{0}$ - середина отрезка $[a-\rho, a]$, тогда положим

$$
J_{1}:=\left\{\cos \left(t-t_{0}\right) \mid t \in[-\pi, \pi] \backslash\left(a-\frac{3 \rho}{2}, a+\frac{3 \rho}{2}\right)\right\}, \quad J_{2}:=\left\{\cos \left(t-t_{0}\right) \mid t \in[a-\rho, a]\right\},
$$

а в качестве $J$ рассмотрим отрезок $[-2,2]$. Тогда существуют число $c, 0<c<1$, и алгебраические многочлены $R_{n \varepsilon}$, удовлетворяющие условиям (2)-(4). Легко видеть, что для тригонометрического многочлена $Q_{n \varepsilon}(t)=R_{n \varepsilon}\left(\cos \left(t-t_{0}\right)\right)$ выполнены условия $(6)-(8)$, где константа $\tau$ возникает при переходе к основанию $e$.

Пусть $g_{\Gamma_{E}}(\zeta, 0)$ и $g_{\Gamma_{E}}(\zeta, \infty)$ - функции Грина области $\overline{\mathbb{C}} \backslash \Gamma_{E}$ с полюсами в точках 0 и $\infty$ соответственно. Из регулярности множества $E$ (а соответственно и $\Gamma_{E}$ ) следует непрерывность $g_{\Gamma_{E}}(\zeta, 0)$ и $g_{\Gamma_{E}}(\zeta, \infty)$ в точках, отличных от 0 и $\infty$, а также их равенство нулю в точках $\Gamma_{E}$. Следовательно, существует $0<\delta<1, \delta=\delta(\tau)$ такое, что если $t \in \mathbb{R}, \operatorname{dist}(t, E) \leqslant \delta$, то

$$
g_{\Gamma_{E}}\left(e^{i t}, 0\right)<\frac{\tau^{2}}{2} .
$$

Выберем $m$ достаточно большим, чтобы для множества $E_{m}^{+}$выполнялось: $\operatorname{dist}(t, E) \leqslant \delta$ для всех $t \in E_{m}^{+}$.

Рассмотрим тригонометрический полином $T_{n} Q_{n \varepsilon}$ степени не выше $n(1+\varepsilon)$. Если $t \in E$, то из (7) вытекает, что

$$
\left|T_{n}(t) Q_{n \varepsilon}(t)\right| \leqslant\left\|T_{n}\right\|_{E} .
$$

Далее нам понадобится неравенство типа Бернштейна-Уолша для $T_{n}$ в точках вещественной оси. Пусть

$$
\begin{aligned}
T_{n}(t) & =\sum_{k=0}^{n}\left(A_{k} \cos k t+B_{k} \sin k t\right) \\
& =\sum_{k=0}^{n}\left(\operatorname{Re} A_{k} \cos k t+\operatorname{Re} B_{k} \sin k t\right)+i \sum_{k=0}^{n}\left(\operatorname{Im} A_{k} \cos k t+\operatorname{Im} B_{k} \sin k t\right) .
\end{aligned}
$$

Рассмотрим алгебраические многочлены

$$
S_{n}^{(1)}(z)=\sum_{k=0}^{n}\left(\operatorname{Re} A_{k}-i \operatorname{Re} B_{k}\right) z^{k}, \quad S_{n}^{(2)}(z)=\sum_{k=0}^{n}\left(\operatorname{Im} A_{k}-i \operatorname{Im} B_{k}\right) z^{k} .
$$


Легко проверяется, что при всех комплексных $t$ имеет место равенство $T_{n}(t)=\eta\left(e^{i t}\right)$, в котором

$$
\eta(z)=\frac{1}{2}\left[S_{n}^{(1)}(z)+\overline{S_{n}^{(1)}\left(\frac{1}{\bar{z}}\right)}\right]+\frac{i}{2}\left[S_{n}^{(2)}(z)+\overline{S_{n}^{(2)}\left(\frac{1}{\bar{z}}\right)}\right] .
$$

Замечая, что $\|\eta\|_{\Gamma_{E}}=\left\|T_{n}\right\|_{E}$, применим к рациональной функции $\eta$ аналог неравенства Бернштейна-Уолша (см. [11; с. 641]), а затем воспользуемся симметрией множества $\overline{\mathbb{C}} \backslash \Gamma_{E}$ относительно единичной окружности:

$$
\left|T_{n}(t)\right|=\left|\eta\left(e^{i t}\right)\right| \leqslant\|\eta\|_{\Gamma_{E}} e^{n\left(g_{\Gamma_{E}}\left(e^{i t}, 0\right)+g_{\Gamma_{E}}\left(e^{i t}, \infty\right)\right)}=\left\|T_{n}\right\|_{E} e^{2 n g_{\Gamma_{E}}\left(e^{i t}, 0\right)}, \quad t \in \mathbb{R} .
$$

Если теперь $t \in E_{m}^{+} \backslash E$, то при достаточно большом $n$ согласно (8) и (9) имеем

$$
\left|T_{n}(t) Q_{n \varepsilon}(t)\right| \leqslant\left\|T_{n}\right\|_{E} \exp \left(2 n g_{\Gamma_{E}}\left(e^{i t}, 0\right)\right) \exp (-n \tau) \leqslant\left\|T_{n}\right\|_{E} \exp \left(n \tau^{2}\right) \exp (-n \tau) \leqslant\left\|T_{n}\right\|_{E}
$$

и, таким образом, $\left\|T_{n} Q_{n \varepsilon}\right\|_{E_{m}^{+}} \leqslant\left\|T_{n}\right\|_{E}$.

Далее, при $t \in[a-\rho, a]$ справедливо неравенство (6), поэтому

$$
\left|\left(T_{n}(t) Q_{n \varepsilon}(t)\right)^{\prime}\right| \geqslant\left|T_{n}^{\prime}(t) Q_{n \varepsilon}(t)\right|-\left|T_{n}(t) Q_{n \varepsilon}^{\prime}(t)\right| \geqslant\left|T_{n}^{\prime}(t)\right|\left(1-e^{-n \tau}\right)-\left|T_{n}(t) Q_{n \varepsilon}^{\prime}(t)\right| .
$$

Используя неравенство Бернштейна для полинома $Q_{n \varepsilon}$ (см. [1; следствие 5.1.5]), получаем $\left\|Q_{n \varepsilon}^{\prime}\right\|_{[-\pi, \pi]} \leqslant n \varepsilon$. Следовательно, для $t \in[a-\rho, a]$ имеем

$$
\left|T_{n}^{\prime}(t)\right|\left(1-e^{-n \tau}\right) \leqslant\left|\left(T_{n}(t) Q_{n \varepsilon}(t)\right)^{\prime}\right|+\left|T_{n}(t) Q_{n \varepsilon}^{\prime}(t)\right| \leqslant\left|\left(T_{n}(t) Q_{n \varepsilon}(t)\right)^{\prime}\right|+n \varepsilon\left\|T_{n}\right\|_{E} .
$$

Отметим теперь, что множество $E_{m}^{+}$состоит из конечного числа отрезков, и мы можем применить соответствующий результат Тотика из [3; теорема 4.1] к $T_{n} Q_{n \varepsilon}$. Таким образом,

$$
\begin{aligned}
\left|T_{n}^{\prime}(t)\right|\left(1-e^{-n \tau}\right) & \leqslant((1+\varepsilon) n)^{2}\left(1+o_{E_{m}^{+}}(1)\right) 8 \pi^{2} \Omega\left(\Gamma_{E_{m}^{+}}, e^{i a}\right)^{2}\left\|T_{n} Q_{n \varepsilon}\right\|_{E_{m}^{+}}+n \varepsilon\left\|T_{n}\right\|_{E} \\
& \leqslant n^{2}\left\|T_{n}\right\|_{E}\left(\left(1+o_{E_{m}^{+}}(1)\right)(1+\varepsilon)^{2} 8 \pi^{2} \Omega\left(\Gamma_{E_{m}^{+}}, e^{i a}\right)^{2}+\frac{\varepsilon}{n}\right)
\end{aligned}
$$

используя монотонность $\Omega(\cdot, a)$, продолжаем оценку

$$
\leqslant n^{2}\left\|T_{n}\right\|_{E}\left(\left(1+o_{E}(1)\right)(1+\varepsilon)^{2} 8 \pi^{2} \Omega\left(\Gamma_{E}, e^{i a}\right)^{2}+\frac{\varepsilon}{n}\right) .
$$

Так как $\varepsilon>0$ было выбрано произвольно, то (5) верно для регулярных компактных множеств $E$.

Условие регулярности можно снять, используя множества $E_{m}^{-}$и $\Gamma_{E_{m}^{-}}$. Действительно,

$$
\left\|T_{n}^{\prime}\right\|_{[a-\rho, a]} \leqslant n^{2}\left\|T_{n}\right\|_{E_{m}^{-}}\left(1+o_{E_{m}^{-}}(1)\right) 8 \pi^{2} \Omega\left(\Gamma_{E}, e^{i a}\right)^{2} \leqslant n^{2}\left\|T_{n}\right\|_{E}\left(1+o_{E_{m}^{-}}(1)\right) 8 \pi^{2} \Omega\left(\Gamma_{E}, e^{i a}\right)^{2},
$$

а так как согласно лемме 1 величина $\Omega\left(\Gamma_{E_{m}^{-}}, e^{i a}\right)$ может быть выбрана сколь угодно близкой к $\Omega\left(\Gamma_{E}, e^{i a}\right)$ при достаточно большом $m$, то (5) справедливо в общем случае.

Покажем теперь точность полученной оценки. Для этого построим последовательность тригонометрических многочленов $\left\{T_{n}\right\}_{n=1}^{\infty}, \operatorname{deg}\left(T_{n}\right)=n$, такую, что

$$
\frac{\left|T_{n}^{\prime}(a)\right|}{n^{2}\left\|T_{n}\right\|_{E}} \rightarrow 8 \pi^{2} \Omega\left(\Gamma_{E}, a\right)^{2}, \quad n \rightarrow \infty .
$$


Как уже было отмечено, множества $E_{m}^{+}$состоят из конечного числа отрезков, поэтому к ним применима теорема 4.1 работы [3], согласно которой существует последовательность тригонометрических многочленов $\left\{T_{m, n}\right\}_{n=1}^{\infty}, \operatorname{deg}\left(T_{m, n}\right)=n$, такая, что

$$
\left|T_{m, n}^{\prime}(a)\right| \geqslant\left(1-o_{E_{m}^{+}}(1)\right) 8 \pi^{2} \Omega\left(\Gamma_{E_{m}^{+}}, e^{i a}\right)^{2} n^{2}\left\|T_{m, n}\right\|_{E_{m}^{+}} .
$$

Так как $E \subset E_{m}^{+}$, то $\left\|T_{m, n}\right\|_{E_{m}^{+}} \geqslant\left\|T_{m, n}\right\|_{E}$, поэтому

$$
\left|T_{m, n}^{\prime}(a)\right| \geqslant\left(1-o_{E_{m}^{+}}(1)\right) 8 \pi^{2} \Omega\left(\Gamma_{E_{m}^{+}}, e^{i a}\right)^{2} n^{2}\left\|T_{m, n}\right\|_{E} .
$$

На этот раз уже величина $\Omega\left(\Gamma_{E_{m}^{+}}, a\right)$ может быть сколь угодно близкой к $\Omega\left(\Gamma_{E}, a\right)$ при достаточно большом $m$, поэтому в качестве искомой последовательности может быть выбрана последовательность $T_{n}=T_{m_{n}, n}$, если $m_{n}$ стремиться к бесконечности достаточно медленно, при $n \rightarrow \infty$.

Если теперь через $H$ обозначить наименьшую дугу на $\mathbb{T}$, соединяющую точки $e^{i(a-\rho)}$ и $e^{i a}$, то имеет место

СледствиЕ. Для алгебрачческого полинома $P_{n}$ справедливо неравенство

$$
\left\|P_{n}^{\prime}\right\|_{H} \leqslant(1+o(1)) n^{2} 2 \pi^{2} \Omega\left(\Gamma_{E}, e^{i a}\right)^{2}\left\|P_{n}\right\|_{\Gamma_{E}} .
$$

Это неравенство точное, так как существует последовательность полиномов $P_{n} \not \equiv 0$, $n=1,2, \ldots$, maкux, что

$$
\left|P_{n}^{\prime}\left(e^{i a}\right)\right| \geqslant(1-o(1)) n^{2} 2 \pi^{2} \Omega\left(\Gamma_{E}, e^{i a}\right)^{2}\left\|P_{n}\right\|_{\Gamma_{E}} .
$$

Величина о(1) равномерно по $P_{n}$ стремится $к$ нулю при $n \rightarrow \infty$.

ДокАзАтельство. Мы можем предполагать, что $n$ - четное (так как $(n+1)^{2} / n^{2}=$ $1+o(1), n \rightarrow \infty)$. Рассмотрим тригонометрический полином $T_{n / 2}=e^{-i t n / 2} P_{n}\left(e^{i t}\right)$ степени выше, чем $n / 2$. Для доказательства (10) достаточно применить теорему 1.

Неравенство (11) доказывается так же, как асимптотическая точность в теореме 1 , только на этот раз, используется соответствующая часть следствия 4.3 работы [3].

\section{СПИСОК ЦИТИРОВАННОЙ ЛИТЕРАТУРЫ}

[1] P. Borwein, T. Erdélyi, Polynomials and Polynomial Inequalities, Grad. Texts in Math., 161, Springer-Verlag, New York, 1995. [2] V. Totik, Acta Math., 187:1 (2001), 139-160. [3] V. Totik, "Bernstein and Markov type inequalities for trigonometric polynomials on general sets", Int. Math. Res. Not. (to appear). [4] B. Nagy, V. Totik, Constr. Approx., 37:2 (2013), 223-232. [5] S. I. Kalmykov, B. Nagy, V. Totik, "Asymptotically Sharp Markov and Schur Inequalities on General Sets", Complex Analysis and Operator Theory, 2014. [6] E. B. Saff, V. Totik, Logarithmic Potentials with External Fields, Grundlehren Math. Wiss., 316. Springer-Verlag, Berlin, 1997. [7] T. Ransford, Potential Theory in the Complex Plane, London Math. Soc. Stud. Texts, 28, Cambridge Univ. Press, Cambridge, 1995. [8] V. Totik, Adv. Math., 252 (2014), 114-149. [9] F. Peherstorfer, R. Steinbauer, SIAM J. Math. Anal., 32:2 (2000), 385-402. [10] A. Ancona, Théorie du potentiel, Lecture Notes in Math., 1096, Springer-Verlag, Berlin, 1984, 34-68. [11] A. А. Гончар, Матем. сб., 78:4 (1969), 640-654.

\section{С. И. Калмыков}

Поступило

Дальневосточный федеральный

10.11.2014

университет, г. Владивосток;

Bolyai Institute, University of Szeged, Венгрия;

Институт прикладной математики

ДВО РАН, г. Владивосток

E-mail: sergeykalmykov@inbox.ru 